\title{
Anti-inflammatory and immunomodulatory effects of Paeonia lactiflora Pall., a traditional Chinese herbal medicine
}

\author{
Dong-Yi He ${ }^{1}$ and Sheng-Ming Dai ${ }^{2 *}$ \\ Department of Rheumatology, Shanghai Guanghua Hospital, Shanghai, China \\ Department of Rheumatology and Immunology, Changhai Hospital, Second Military Medical University, Shanghai, China
}

Edited by:

Yili Yang, Second Military Medical

University, China

\section{Reviewed by:}

Yili Yang, Second Military Medical

University, China

Genbin Shi, National Cancer institute, USA

\section{*Correspondence.}

Sheng-Ming Dai, Department of

Rheumatology and Immunology,

Changhai Hospital, Second Military

Medical University, 174 Changhai Road,

Shanghai 200433, China.

e-mail:dsm@medmail.com.cn
In China, Korea, and Japan, a decoction of the dried root without bark of Paeonia lactiflora Pall. has been used in the treatment of rheumatoid arthritis, systemic lupus erythematosus, hepatitis, dysmenorrhea, muscle cramping and spasms, and fever for more than 1200 years. A water/ ethanol extract of the root is now known as total glucosides of peony (TGP), which contains more than 15 components. Paeoniflorin is the most abundant ingredient and accounts for the pharmacological effects observed with TGP in both in vitro and in vivo studies. The analgesic effect of TGP was confirmed in various animal models of pain, which may be mediated partly by adenosine A1 receptor. The direct anti-inflammatory effects of TGP were observed in animal models of both acute and subacute inflammation, by inhibiting the production of prostaglandin E2, leukotriene B4, and nitric oxide, and by suppressing the increase of intracellular calcium ion concentration. TGP was also reported to have protective effects of cells against oxidative stress. In vitro, dual effects of TGP were noted on the proliferation of lymphocytes, differentiation of $\mathrm{Th} / \mathrm{Ts}$ lymphocytes, and the production of proinflammatory cytokines and antibodies. In vivo, TGP inhibited the delayed-type hypersensitivity in immuno-activated mice, and enhanced the delayed-type hypersensitivity in immuno-suppressed mice. In adjuvant arthritis rats, paeoniflorin exerted immunosuppressive effects. The beneficial effects of TGP in treating rheumatoid arthritis were verified by randomized controlled trials. The adverse events of TGP were mainly gastrointestinal tract disturbances, mostly mild diarrhea.

Keywords: Paeoniae Radix, anti-inflammation, immunomodulation, Chinese herbal medicine
Paeoniae Radix is one of the most well-known herbs in China, Korea, and Japan for more than 1200 years. Paeonies are divided into two groups: the tree Peony (also named Paeonia Moutan) and the herbaceous kinds. Paeonia lactiflora Pall. (also named Chinese Peony) is a herbaceous perennial flowering plant in the family Paeoniaceae with fleshy roots and annual stems. It is about $60-100 \mathrm{~cm}$ tall with large compound leaves $20-40 \mathrm{~cm}$ long. The flower buds are large and round, opening into large flowers $8-16 \mathrm{~cm}$ diameter, with 5-10 white, pink, or crimson petals and yellow stamens (Figure 1). It is native to east Asia, and is grown on dry open stony slopes, riverbanks and sparse woodland edges.

The dried root without bark of P. lactiflora Pall., namely Radix Paeoniae Alba, has been used as a medicinal herb in traditional Chinese medicine for centuries, where it is called Bái Sháo (literally: "White Peony"). The root is dug in summer or autumn from cultivated plants that are 4-5 years old, and cleaned with water. After removal of the bark and rootlets, it is boiled in water for a short while, dried in the sun, and then sliced. A decoction of the root has been used in the treatment of rheumatoid arthritis, systemic lupus erythematosus, hepatitis, dysmenorrhea, muscle cramping and spasms, and fever with a long history.

A water/ethanol extract of Radix Paeoniae Alba is known as total glucosides of peony (TGP), which contains more than 15 components, including paeoniflorin, albiflorin, oxypaeoniflorin, benzoylpaeoniflorin, oxybenzoyl-paeoniflorin, paeoniflorigenone, lactiflorin, galloylpaeoniflorin, paeonin, paeonolide, and paeonol
(Zhang et al., 2001; Tan et al., 2010). Most of them are monoterpene glucosides, and their structures are shown in Figure 2. Among them, paeoniflorin $\left(\mathrm{C}_{23} \mathrm{H}_{28} \mathrm{O}_{11}\right.$, with a molecular weight of 480.45 ), a water-soluble compound, is the most abundant (>90\%) and accounts for the pharmacological effects observed with TGP in both in vitro and in vivo studies. So the content of paeoniflorin is used for the standardization of the dosage of TGP. A preparation of TGP was approved by State Food and Drug Administration of China to enter market as a disease-modifying drug for rheumatoid arthritis in 1998. In recent years, a lot of studies describing the chemistry and the pharmacology of TGP have been published, but often in Chinese. Here, we briefly reviewed the immunomodulatory and anti-inflammatory effects of TGP.

\section{ANALGESIC EFFECT OF TGP}

Although the root of $P$. lactiflora Pall. was believed to be effective in relieving various pain in traditional Chinese medicine, the direct evidence was not obtained in modern medicine until 1988 (Wang et al., 1988). TGP suppressed writhing induced by intraperitoneal injection of acetic acid, squeak induced by footpad stimulation with electricity, and hot-plate reaction in mice and/or rats in a dose-dependent manner. Naloxone failed to abolish these effects of TGP. These data verified the analgesic effect of TGP in various animal models of pain, and suggest the effect is not mediated by the opiate receptors. The analgesic effect of TGP was further confirmed by subsequent studies (Gao et al., 2002; Lee et al., 2008; 

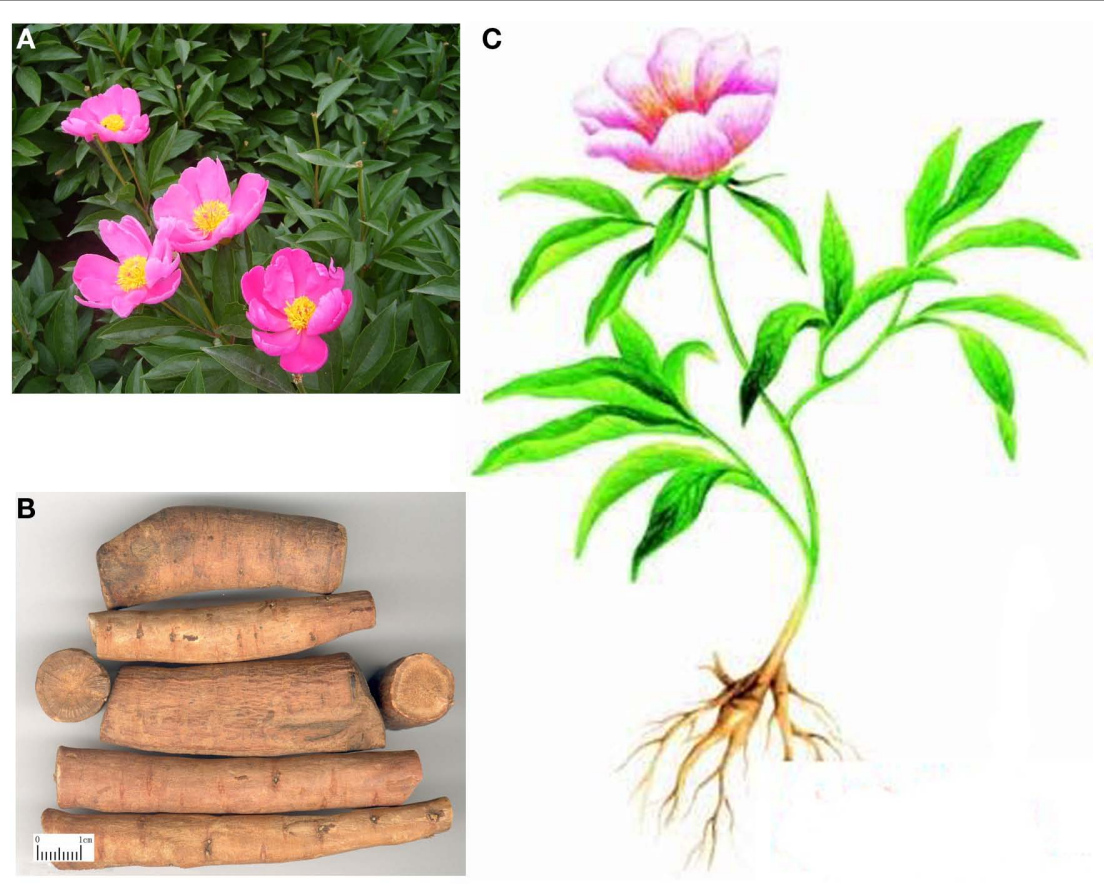

FIGURE 1 | Paeonia lactiflora Pall. (A) overground part of the plant; (B) root; (C) scheme of an intact plant.

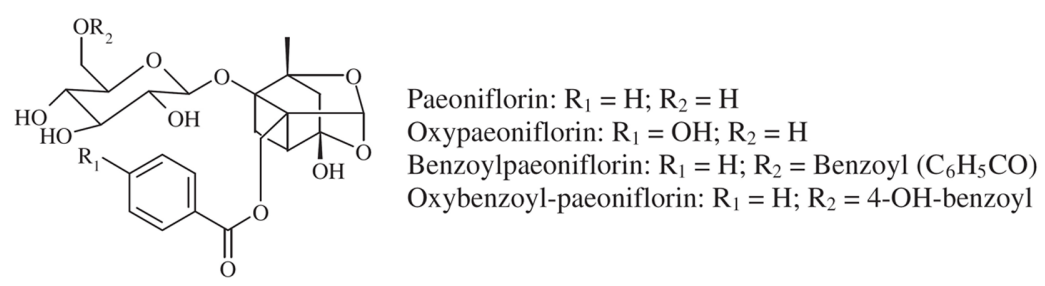

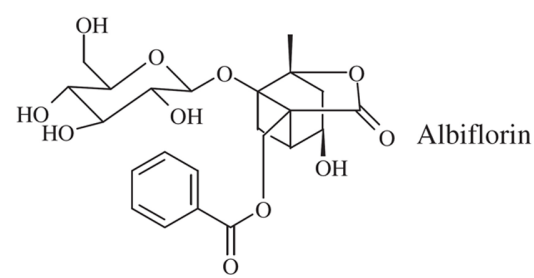

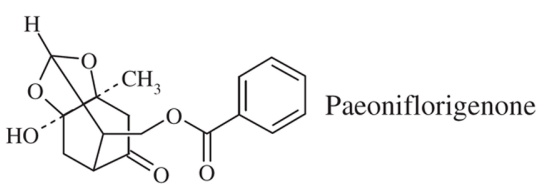

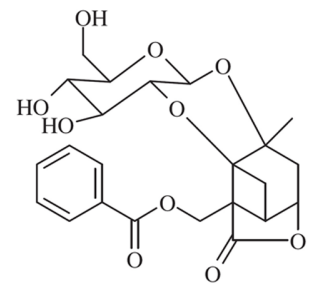

Lactiflorin

FIGURE 2 | Chemical structures of main components in total glucosides of peony.

Ou-Yang, 2008). Recently, it was demonstrated that paeoniflorin, a chief active ingredient in the root of $P$. lactiflora Pall., is effective in relieving colorectal distention (CRD)-induced visceral pain in rats with visceral hyperalgesia induced by neonatal maternal separation. Furthermore, analgesic effect of paeoniflorin is mediated, at least in part, by adenosine A1 receptor (Zhang et al., 2009).

\section{ANTI-INFLAMMATORY EFFECT OF TGP}

The direct anti-inflammatory effects of TGP were observed in animal models of both acute and subacute inflammation, such as dimethylbenzene-induced ear swelling, carrageenan-induced paw edema, subcutaneous tampon-induced granuloma, complete Freund's adjuvant-induced arthritis, and collagen-induced 
arthritis (Gao et al., 2002; Zheng and Wei, 2005; Zhu et al., 2005; Xu et al., 2007; Ou-Yang, 2008). TGP also decreased the permeability of peritoneal capillary which was enhanced by acetic acid (Ou-Yang, 2008). Prostaglandin E2 (PGE2) is synthesized in substantial amounts at sites of inflammation where it acts as a potent vasodilator and causes an increase in vascular permeability and edema synergistically with other mediators. Leukotriene B4 (LTB4) is chemotactic for neutrophils and is important in the characteristic infiltration of these cells in the acute inflammatory response. PGE2 enhances the chemotactic activity of LTB4. TGP $(0.001 \sim 100 \mathrm{mg} / \mathrm{L})$ inhibited the production of LTB4 from peritoneal macrophages and the production of PGE2 from calcimycin A23187-stimulated macrophages in a dose-dependent manner (Li et al., 1992, 1994). The A23187-induced increase of intracellular calcium ion concentration $\left[\left(\mathrm{Ca}^{2+}\right)_{i}\right]$ in macrophages was also inhibited by TGP ( $\mathrm{Li}$ et al., 1994). Inflammation induces oxidative stress by producing oxidants like reactive oxygen species and nitric oxide (NO). $\mathrm{NO}$ is also a potent mediator of inflammation. TGP markedly suppressed lipopolysaccharide (LPS)-induced NO production and inducible nitric oxide synthase (iNOS) expression in rat peritoneal macrophages (Chen et al., 2008). In addition, the production of reactive oxygen species from LPS-stimulated macrophages was inhibited by higher concentrations $(11.25 \sim 125 \mathrm{mg} / \mathrm{L})$, but enhanced by lower concentrations $(0.09 \sim 11.25 \mathrm{mg} / \mathrm{L})$ of TGP in a dose-dependent manner (Liang et al., 1990). In vivo studies also showed that TGP inhibited the production of PGE2 by macrophages in rats with adjuvant arthritis (Li et al., 1994). Recently, paeoniflorin, the principle component of TGP, was also demonstrated to be effective in inhibiting the production of NO and PGE2 from LPS-stimulated RAW 264.7 macrophages (Kim and $\mathrm{Ha}, 2009$ ). An in vivo study showed that both TGP and paeoniflorin potently inhibited passive cutaneous anaphylaxis reaction induced by IgE-antigen complex and scratching behaviors induced by compound $48 / 80$ in mice (Lee et al., 2008). Pretreatment with paeoniflorin decreased mortality, reduced lung and kidney injury, decreased serum creatinine level, and improve systolic function of heart in mice challenged with LPS. Further experiments showed that paeoniflorin inhibited LPS-stimulated tumor necrosis factor- $\alpha$ (TNF- $\alpha$ ) and interleukin (IL)- $1 \beta$ release and promoted LPS-induced IL-10 production (Cao et al., 2011).

All together these data indicate that TGP exerts anti-inflammatory effects by inhibiting the production of inflammatory mediators, such as PGE2, LTB4, NO, reactive oxygen species, and proinflammatory cytokines.

\section{IMMUNOMODULATORY EFFECTS OF TGP DUAL EFFECTS ON PROLIFERATION AND APOPTOSIS OF LYMPHOCYTES}

In vitro, dual effects of TGP on concanavalin A (ConA)-induced proliferation of mouse splenocytes were noted (Wang et al., 1991). TGP enhanced the proliferation at lower concentrations $(0.05 \sim 0.4 \mathrm{mg} / \mathrm{L})$ but inhibited the proliferation at higher concentrations $(0.4 \sim 1.6 \mathrm{mg} / \mathrm{L})$ in a dose-dependent manner. The dual effects of TGP on the proliferation of lymphocytes were confirmed by other studies (Li et al., 1990; Wang et al., 1992). In addition, TGP upregulated the differentiation of both helper $\mathrm{T}$ cells (Th) and suppressor T cells (Ts), and reversed the inhibitory effect of cyclosporine A on Th differentiation and the inhibitory effect of levamisole on Ts differentiation. TGP increased the ratio of Th/ Ts at a lower concentration $(0.2 \mathrm{mg} / \mathrm{L})$, but decreased the ratio of Th/Ts at a higher concentration $(6.0 \mathrm{mg} / \mathrm{L}$; Wang et al., 1992). In vivo studies also confirmed above findings. TGP inhibited the delayed-type hypersensitivity (allergic contact dermatitis induced by 2,4-dinitrofluorobenzene) in immuno-activated mice by downregulation of Th/Ts ratio and serum level of TNF- $\alpha$, and enhanced the delayed-type hypersensitivity in immuno-suppressed mice by upregulation of Th/Ts ratio (Wang et al., 1990; Chen et al., 2010). In adjuvant arthritis rats, paeoniflorin significantly inhibited the proliferation of mesenteric lymph node lymphocytes (Wu et al., 2007). In addition, paeoniflorin induces apoptosis in murine $T$ lymphocytes and Jurkat human $\mathrm{T}$ cell leukemia cells through a redox-linked mechanism (Tsuboi et al., 2004). These data suggest TGP may suppress over-activated immuno-responses by inhibiting the proliferation of lymphocytes, balancing the differentiation of Th and Ts cells, and inducing the apoptosis of lymphocytes.

\section{DUAL EFFECTS ON PRODUCTION OF PROINFLAMMATORY CYTOKINES}

In vitro, dual effects of TGP on IL-1 and IL-2 production were also noted (Wei et al., 1989; Li et al., 1990). TGP enhanced IL-1 production by LPS-stimulated mouse splenocytes at lower concentrations $(0.5 \sim 12.5 \mathrm{mg} / \mathrm{L})$ but inhibited the production at higher concentrations $(12.5 \sim 312.5 \mathrm{mg} / \mathrm{L}$ ) in a dose-dependent manner (Li et al., 1990). TGP enhanced IL-1 production by LPS-stimulated macrophages, and IL-2 production by ConA-stimulated mouse splenocytes at lower concentrations $(0.5 \sim 12.5 \mathrm{mg} / \mathrm{L})$. However, it inhibited the production at higher concentrations $(12.5 \sim 62.5 \mathrm{mg} / \mathrm{L}$; Wei et al., 1989; Liang et al., 1990). In rats with experimental colitis, serum levels of IL-6, IL-17, and IL-23 were lowered, and the colonic expression levels of transforming growth factor- $\beta 1$ (TGF- $\beta 1$ ) and Foxp3 were elevated by treatment with TGP (Wang et al., 2010). In adjuvant arthritis rats, paeoniflorin significantly downregulated the level of proinflammatory cytokine IL-2, and upregulated the levels of IL-4 and TGF- $\beta 1$ (Wu et al., 2007). These data suggest that paeoniflorin exerts the anti-inflammatory and immunomodulatory effects by balancing the function of Th1 cells and Th2 cells.

\section{DUAL EFFECTS ON PRODUCTION OF ANTIBODIES}

In vitro, dual effects of TGP on IgM-antibody production by mouse splenocytes immunized with red blood cells of sheep were found (Wang et al., 1991, 1992). TGP enhanced the production of IgMantibodies at lower concentrations $(0.1 \sim 0.4 \mathrm{mg} / \mathrm{L})$ but inhibited the production at higher concentrations $(0.4 \sim 3.2 \mathrm{mg} / \mathrm{L})$. However, the concentration of antibodies to type II collagen in rats with collagen-induced arthritis was not changed by TGP treatment, in spite of the improvements in the hind paw swelling and the arthritis scores (Wang et al., 2011).

\section{ANTIOXIDANT EFFECT OF TGP}

TGP was also reported to have protective effects of cells against oxidative stress. Kim et al. (2009) found that TGP significantly protected primary cultures of rat cortical cells exposed to oxidative stress induced by $\mathrm{H}_{2} \mathrm{O}_{2}$. Paeoniflorin was reported to exert a neuroprotective effect on glutamate-induced neurotoxicity in PC12 cells, via inhibiting oxidative stress and $\mathrm{Ca}^{2+}$ overload (Mao et al., 2010). The antioxidative effect of paeoniflorin on the LPS-induced 
liver inflammatory reactions and on diabetes-associated renal damage were also demonstrated by in vivo studies (Kim and Ha, 2010; Su et al., 2010).

\section{THERAPEUTIC USAGE OF TGP}

The anti-inflammatory and immunomodulatory effects of TGP provide the therapeutic basis in the treatment of autoimmune diseases, such as rheumatoid arthritis and systemic lupus erythematosus. There have been numerous clinical reports on the efficacy and safety of TGP in various diseases. Unfortunately, few of them have been substantiated by randomized controlled trials with large sample size. The first prospective, double-blind trial of TGP was conducted in 1993, and 450 patients with rheumatoid arthritis were randomized to receive TGP ( $1.8 \mathrm{~g} /$ day $)$ or methotrexate $(10 \mathrm{mg} /$ week) for 12 weeks. Therapeutic response was achieved in $71.7 \%$ of TGP-treated patients and $81.7 \%$ of methotrexate-treated patients, respectively. The adverse events of TGP occurred in $13.3 \%$ patients, and most of them were gastrointestinal tract disturbances, mostly mild diarrhea. Most of the events were self-resolved in 1-2 weeks. No adverse events on hepatic, renal or hematological tests were found. The similar results were confirmed in a phase III clinical trial of 1016 patients with rheumatoid arthritis (Zhou and Li, 2003). Some pilot studies also showed therapeutic benefits of

\section{REFERENCES}

Cao, W., Zhang, W., Liu, J., Wang, Y., Peng, X., Lu, D., Qi, R., Wang, Y., and Wang, H. (2011). Paeoniflorin improves survival in LPS-challenged mice through the suppression of TNF- $\alpha$ and IL- $1 \beta$ release and augmentation of IL-10 production. Int. Immunopharmacol. 11, 172-178.

Chen, F., Zhu, Z., Gao, H., and Luo, D. (2010). Effects of total glucosides of paeony on murine allergic contact dermatitis. Shi Yong Lin Chuang Yi Yao Za Zhi 14, 5-7, 16 (in Chinese).

Chen, G., Guo, L. X., Deng, X. H., Yin, Z. Y., and Jing, J. J. (2008). Effects of total glucosides of paeony on nitric oxide and inducible nitric oxide synthase production in macrophages and its mechanism. Zhongguo Mian Yi Xue Za Zhi24, 345-347, 351 (in Chinese).

Gao, C., Wu, Y., Wang, Y., Yan, J., Pan, H., Cao, L., and Liang, R. (2002). AntiInflammatory and analgesic effects of total glucosides of paeonia injection. Zhong Yao Xin Yao Yu Lin Chuang Yao Li 13, 163-165 (in Chinese).

Kim, I. D., and Ha, B. J. (2009). Paeoniflorin protects RAW $264.7 \mathrm{mac}$ rophages from LPS-induced cytotoxicity and genotoxicity. Toxicol In Vitro 23, 1014-1019.

Kim, I. D., and Ha, B. J. (2010). The effects of paeoniflorin on LPS-induced liver inflammatory reactions. Arch. Pharm. Res. 33, 959-966.

Kim, S. H., Lee, M. K., Lee, K. Y., Sung, S. H., Kim, J., and Kim, Y. C. (2009). Chemical constituents isolated from
Paeonia lactiflora roots and their neuroprotective activity against oxidative stress in vitro. J. Enzyme Inhib. Med. Chem. 24, 1138-1140.

Lee, B., Shin, Y. W., Bae, E. A., Han, S. J., Kim, J. S., Kang, S. S., and Kim, D. H. (2008). Antiallergic effect of the root of Paeonia lactiflora and its constituents paeoniflorin and paeonol. Arch. Pharm. Res. 31, 445-450.

Li, J., Chen, M. Z., and Xu, S. Y. (1994). Effect of total glucosides of paeony on prostaglandin E2 derived from peritoneal macrophages and related mechanisms in rats. Zhongguo Yao Li Xue Tong Bao 10, 267-270 (in Chinese).

Li, J., Liang, J. S., Zhou, A. W., Chen, M. Z., and Xu, S. Y. (1990). Modulatory effects of total glucosides of paeony on B lymphocyte proliferation and interleukin 1 production. Zhongguo Yao Li Xue Yu Du Li Xue Za Zhi 4, 53-55 (in Chinese).

Li, J., Zhao, W., Chen, M., and Xu, S. (1992). Effect of total glucosides of paeony on leukotriene B4 by peritoneal macrophages in rats. Zhongguo Yao Li Xue Tong Bao 8, 36-39 (in Chinese).

Liang, J. S., Chen, M. Z., and Xu, S. Y. (1990). Effect of total glucosides of paeony on the function of peritoneal macrophages in rats. Zhongguo Yao Li Xие Yu Du Li Xue Za Zhi 4, 153-154 (in Chinese).

Long, J. W., Wang, Y. Y., Pi, X. M., and Tu, Y. T. (2010). Clinical observation on the treatment of chronic urticaria

TGP in systemic lupus erythematosus (Shuai et al., 2003), Sjögren syndrome (Lu et al., 2006), ankylosing spondylitis (Wang et al., 2007), and chronic urticaria (Long et al., 2010).

\section{CONCLUDING REMARKS}

A water/ethanol extract of the dried root without bark of P. lactiflora Pall. is now known as TGP, and paeoniflorin is the most abundant component (>90\%) of TGP. TGP is effective to relieve the pain in various animal models. TGP also inhibits both acute and subacute inflammation by suppressing the increase of intracellular calcium ion concentration, and inhibiting the production of inflammatory mediators, such as PGE2, LTB4, and NO. There are dual effects of TGP on the proliferation of lymphocytes, differentiation of $\mathrm{Th} / \mathrm{Ts}$ lymphocytes, and production of proinflammatory cytokines and IgM-antibodies, which depend on the activity of immuno-responses. The underlying mechanisms of TGP responsible for the unique immunomodulatory effects need to be further studied.

\section{ACKNOWLEDGMENT}

This study was supported in part by the grants from National Natural Science Foundation of China (No. 30972729) and National Science and Technology Major Project of China (No. 2009ZX09303-002).

with total glucosides of paeony capsule combined with cetirizine. Chin J. Integr. Med. 16, 353n-366n.

Lu, J., Yang, P., Shen, H., Xiao, W., and Zhao,L. (2006). Clinical application of total glucosides of paeony in Sjögren syndrome. Zhongguo Yi Ke DaXueXue Bao 35, 522-524 (in Chinese).

Mao, Q. Q., Zhong, X. M., Li, Z. Y., and Huang,Z. (2010). Paeoniflorin protects against NMDA-induced neurotoxicity in PC12 cells via Ca2+ antagonism. Phytother. Res. doi: 10.1002/ptr.3321. [Epub ahead of print].

Ou-Yang, Y. (2008). Research of alcohol extract of Paeonia lactiflora Pall in anti-inflammatory and analgesic effect. Shu Li Yi Yao Za Zhi21, 600-602 (in Chinese).

Shuai, Z. W., Xu, J. H., Liu, S., Wei, W., $\mathrm{Xu}$, S. Q., and Xu, S. Y. (2003). Clinical observation of total glucosides of paeony in the treatment of systemic lupus erythematosus. Zhongguo Zhong Xi Yi Jie He Za Zhi23, 188-191 (in Chinese).

Su, J., Zhang, P., Zhang, J. J., Qi, X. M., Wu, Y. G., and Shen, J. J. (2010). Effects of total glucosides of paeony on oxidative stress in the kidney from diabetic rats. Phytomedicine 17, 254-260.

Tan, J., Zhao, Q., Yang, L., Shang, Z., Du, Z., and Yan, M. (2010). Chemical constituents in roots of Paeonia lactiflora. Zhong Cao Yao 41, 1245-1248 (in Chinese).

Tsuboi, H., Hossain, K., Akhand, A. A., Takeda, K., Du, J., Rifa'i, M., Dai, Y., Hayakawa, A., Suzuki, H., and Nakashima, I. (2004). Paeoniflorin induces apoptosis of lymphocytes through a redox-linked mechanism. J. Cell. Biochem. 93, 162-172.

Wang, S., Wang, J., Bian, H., Wang, H., and Li, P. (2007). Clinical observation on total glucosides of paeony combined with sulfasalazine in treatment of ankylosing spondylitis. Zhongguo Zhong XiY Jie He Za Zhi 27, 217-219 (in Chinese).

Wang, X. W., Cheng, M. Z., and Xu, S. Y. (1991). Effects of total glucosides of paeony on immune system. Zhongguo Bing Li Sheng Li Za Zhi 7, 609-611 (in Chinese).

Wang, X. W., Chen, M. Z., and Xu, S. Y. (1992). The effects of total glucosides' of paeony (TGP) on T lymphocyte subsets. Zhongguo Yao Li Xue Tong Bao 8, 340-343, 314 (in Chinese).

Wang, X. W., Wei, W., Chen, M. Z., and Xu, S.Y. (1990). Mechanisms of immunomodulatory actions of total glucosides of paeonyin mice. Zhongguo Yao Li Xue Tong Bao 6, 363-366 (in Chinese).

Wang, Y., Chen, M., and Xu, S. (1988). Analgesic effect of total glucosides of Paeonia lactiflora. Zhongguo Yao Li Xue Yu Du Li Xue Za Zhi 2, 6-10 (in Chinese).

Wang, Z., Wu, Z. X., Yang, J. H., Yang, F., and $\mathrm{Wu}, \mathrm{Q}$. (2010). Total glucosides of paeony mitigate trinitrobenzene sulfonic acid-induced experimental colitis in rats. Shijie Hua Ren Xiao Hua Za Zhi 18, 84-88 (in Chinese).

Wang, Q. T., Zhang, L. L., Wu, H. X. and Wei, W. (2011). The expression change of $\beta$-arrestins in fibroblastlike synoviocytes from rats with collagen-induced arthritis and the 
effect of total glucosides of paeony. J. Ethnopharmacol. 133, 511-516.

Wei, W., Liang, J. S., Zhou, A. W., Chen, M. Z., and Xu, S. Y. (1989). Effects of total glucosides of paeony on interleukin 2 production. Zhongguo Yao Li Xue Tong Bao 5, 176-179 (in Chinese).

Wu, H., Wei, W.,Song,L.,Zhang, L., Chen, Y., and Hu,X. (2007).Paeoniflorin induced immune tolerance of mesentericlymph node lymphocytes via enhancing beta2-adrenergic receptor desensitization in rats with adjuvant arthritis. Int. Immunopharmacol. 7, 662-673.

Xu, H. M., Wei, W., Jia, X. Y., Chang, Y., and Zhang, L. (2007). Effects and mechanisms of total glucosides of paeony on adjuvant arthritis in rats. J. Ethnopharmacol. 109, 442-448.
Zhang, X., Wang, J., and Li, X. (2001). A study on the chemical constituents of Paeonia lactiflora Pall. Shengyang Yao Ke Da Xue Xue Bao 18, 30-2 (in Chinese).

Zhang, X. J., Chen, H. L., Li, Z., Zhang, H. Q., Xu, H. X., Sung, J., and Bian, Z. $X$. (2009). Analgesic effect of paeoniflorin in rats with neonatal maternal separation-induced visceral hyperalgesia is mediated through adenosine Al receptor by inhibiting the extracellular signal-regulated protein kinase (ERK) pathway. Pharmacol. Biochem. Behav. 94, 88-97.

Zheng, Y. Q., and Wei, W. (2005). Total glucosides of paeony suppressed adjuvant arthritis in rats and intervening cytokine-signaling between different types of synoviocytes. Int. Immunopharmacol. 5, 1560-1573.

Zhou,Q., and Li,Z.G. (2003).Pharmacology and therapeutic usages of total glucosides of paeony in autoimmune diseases. Zhongguo Xin Yao Yu Lin ChuangZa Zhi 22, 687-691 (in Chinese).

Zhu, L., Wei, W., Zheng, Y. Q., and Jia, X Y. (2005). Effects and mechanisms of total glucosides of paeony on joint damage in rat collagen-induced arthritis. Inflamm. Res. 54, 211-220.

Conflict of Interest Statement: The authors declare that the research was conducted in the absence of any commercial or financial relationships that could be construed as a potential conflict of interest.
Received: 28 December 2010; accepted: 14 February 2011; published online: 25 February 2011.

Citation: He D-Y and Dai S-M (2011) Anti-inflammatory and immunomodulatory effects of Paeonia lactiflora Pall., a traditional Chinese herbal medicine. Front. Pharmacol. 2:10. doi: 10.3389/ fphar.2011.00010

This article was submitted to Frontiers in Ethnopharmacology, a specialty of Frontiers in Pharmacology.

Copyright $\odot 2011$ He and Dai. This is an open-access article subject to an exclusive license agreement between the authors and Frontiers Media SA, which permits unrestricted use, distribution, and reproduction in any medium, provided the original authors and source are credited. 